\title{
EARLY MORTALITY \& MORBIDITY IN MITRAL VALVE REPLACEMENT WITH TOTAL SUB-VALVULAR APPARATUS PRESERVATION, PARTIAL PRESERVATION AND NO PRESERVATION - A COMPARATIVE STUDY
}

\author{
Muhammad Ashfaq, Nasir Ali, Asif Mahmood Janjua, Naser Ali Khan, Muhammad Imran Ashgar, Kifayat Ullah, \\ Yasir Javed
}

Armed Forces Institute of Cardiology/National Institute of Heart Disease (AFIC/NIHD)/National University of Medical Sciences (NUMS) Rawalpindi Pakistan

\begin{abstract}
Objective: To compare the early morbidity \& mortality of total sub-valvular apparatus preservation technique vs partial preservation technique and no preservation technique in mitral valve replacement.

Study Design: Cross-sectional comparative study.

Place and Duration of Study: Department of Cardiac Surgery, Armed Forces Institute of Cardiology Rawalpindi, from Jan 2017 to Dec 2018.

Methodology: One hundred fifty seven patients who underwent Mitral valve replacement were included in study. These patients were divided into three groups; group " $\mathrm{A}$ " comprised of those patients with total subvalvular apparatus preservation $(\mathrm{n} 1=46)$, group " $\mathrm{B}$ " comprised of those patients with partial sub-valvular apparatus preservation $(\mathrm{n} 2=70)$, group " $\mathrm{C}$ " comprised of those patients with no sub-valvular apparatus preservation (n3=41).

Results: There were no statistical difference in the pre-operative data of the patients. No statistical difference noted in cross clamp \& bypass time $(p>0.05)$. The early morbidity in terms of ICU stay, Ventilation time, Inotropic supports, post-operative bleeding, Blood transfusion, postoperative rhythm abnormality \& Re-exploration were statically significant $(p<0.05)$. The mortality rate of patients with total sub-valvular apparatus preservation was $2.17 \%$ which is significantly less than those with partial preservation $(2.85 \%)$ \& no preservation $(9.75 \%)$.

Conclusions: Mitral valve replacement with total subvalvular apparatus preservation had low postoperative morbidity \& mortality in comparison with partial and no preservation techniques.
\end{abstract}

Keywords: Mitral Valve Replacement, Subvalvular Apparatus preservation, Operative Techniques.

This is an Open Access article distributed under the terms of the Creative Commons Attribution License (http://creativecommons.org/licenses/by/4.0), which permits unrestricted use, distribution, and reproduction in any medium, provided the original work is properly cited.

\section{INTRODUCTION}

Mitral valve is a complex anatomical structure which not only plays an important role in maintaining the left ventricular functions but also effects the right ventricular functions as well ${ }^{1}$. Mitral Valve Replacement started in 1961, where Starr-Edward Prosthetic valve was used after excision of the native valve leaflets along with chordae tendineae \& papillary heads, leading to drastic early complication of low cardiac output $\&$ ultimately death ${ }^{2}$. Therefore, the concept of sub-valvular apparatus preservation was introduced. The components of sub-valvular apparatus are ventricular free wall, papillary muscle \& chordae tendineae. Significance of sub-valvular

Correspondence: Dr Muhammad Ashfaq, Adult Cardiac Surgery, AFIC/NIHD Rawalpindi Pakistan apparatus during ventricular systole is to prevent mitral leaflets prolapse in left atrium and improving the left ventricular contraction; process called as annulo-ventricular continuity ${ }^{3}$. In recent past its established that the effective modality of treatment in mitral valve surgery is valve repair with preservation of its sub-valvular apparatus 4 . In certain condition where Mitral valve replacement (MVR) is inevitable because of anatomical \& etiological reasons ${ }^{5}$, different techniques of preservation were developed to restore Left ventricular functions and Atrioventricular anatomy ${ }^{6}$. Some surgeons are against the concept of preservation because of grossly calcific valves, fibrosis of leaflets \& papillary heads, small sized left ventricle \& placement of smaller prosthetic valve ${ }^{1}$. But Its proven from different studies that destruction of Atrioventricular connections leads to early 
heart failure because Left ventricle adopts spherical shape causing poor contraction \& low cardiac output with high mortality 7 . Gomes adopted preservation of both leaflets preservation technique along with the sub-valvular apparatus ${ }^{8}$. Al Saddique reported two main drawbacks of total preservation that were left ventricle outflow obstruction due to extensive tissue and better application of technique in mitral regurgitation patients 9 . To encounter this difficulty Puig et al introduced division of anterior \& posterior leaflets into hemileaflets via trimming of the extra tissue ${ }^{10}$. Keeping in view the importance of chordal preservation, the current study compared the early morbidity \& mortality in MVR with total sub-valvular apparatus preservation technique vs partial preservation technique and no preservation technique.

To compare the early morbidity \& mortality of total sub-valvular apparatus preservation technique vs partial preservation technique and no preservation technique inmitral valve replacement.

\section{METHODOLOGY}

A cross-sectional comparative study was conducted in the Department of Cardiac Surgery, Armed Forces Institute of Cardiology-National Institute of Heart Diseases (AFIC-NIHD), Rawalpindi. Data of patients who underwent MVR from $1^{\text {st }}$ January 2017 to $31^{\text {st }}$ December 2018 was collected from the institutional database. Patients of any gender with age ranging from 20-80 years \& isolated mitral valve disease predominantly Mitral Stenosis was included in the study while patients with multiple valve disease, redo cases, mitral valve disease with coronary artery disease, left atrial size $>60 \mathrm{~mm}$, infective endocarditis, moderate-severe pulmonary hypertension \& BMI $<18$ were excluded from the study sample. Finally, 157 patients who fulfilled the inclusion criteria was included in the study. These patients were divided into three groups; group " $\mathrm{A}$ " comprised of those patients with total sub-valvular apparatus preservation (n1=46), group “ $B$ " comprised of those patients with partial sub-valvular apparatus preservation $(\mathrm{n} 2=70)$, group " $\mathrm{C}$ " comprised of those patients with no sub-valvular apparatus preservation (n3=41).

Total Sub-valvular apparatus preservation technique: In this technique the anterior mitral leaflet (AML) was cut in the center at least $2-3 \mathrm{~mm}$ away from the annulus. The excised leaflet is trimmed in such a way to make two buttons like tissues, connected with the main chordae tendineae. The preserved tissue of AML is stitched with the mitral annulus by pledgeted Ethibond 2/0 at anterolateral \& posteromedial positions. Posterior leaflet is either trimmed or left as such if not hindering the implantation of appropriate size mitral prosthesis.

Partial sub-valvular apparatus preservation technique: In this technique the AML is excised completely along with the sub-valvular apparatus while the posterior mitral leaflet (PML) along with the sub-valvular apparatus is preserved as such. In case of calcific thickened posterior leaflet, it was either trimmed or incised in such a way that two heads were made which was stitched to the annulus with pledgeted Ethibond 2/0.

No preservation technique: In this approach both the AML \& PML along with the sub-valvular apparatus was excised completely without any preservation.

Standard operative technique was adopted for all the patients which included median sternotomy, cardiopulmonary bypass with moderate hypothermia (320C) \& intermittent antegrade blood cardioplegia. Approach to Mitral valve was either through Left atrium or Right atrium-trans septal approach. Technique used for prosthetic mitral valve implantation was either through continuous or interrupted suturing. During the procedure it was ensured that no leaflet or subvalvular tissue interfere in the valve function by checking the movements of the valve disc. The decision regarding the preservation techniques was taken intra-operatively by visualization of the valve anatomy. Demographic data like age, gender, body mass index (BMI), pre-operative ejection fraction and comorbidities were recorded. Peri-operative data of cardiopulmonary 
bypass time \& cross clamp time was noted. Post operatively the ventilation time, Intensive care unit (ICU) stay, bleeding, arrhythmias \& number of deaths were also recorded. Early morbidity \& mortality were recoded till 7th post-operative day. Outcome variables were analyzed in order to determine the mortality and morbidity in each group. Primary outcome in our study was mortality and secondary outcomes were ICU stay, ventilation time, inotropic support \& post-operative new onset of Atrial Fibrillation.

\section{RESULTS}

The demographic profile of the patient is tabulated in table-I. No statistically significant operative Ejection Fraction \& Rhythm was also found to be insignificant.

There was no significant difference noted in peri-operative cardiopulmonary bypass time, cross clamp time as shown in table-II. Statistically significant difference noted in the mitral valve prosthesis size $(p=0.010)$.

There was significant difference recorded in ventilation time \& intensive care unit stay, which is less in group A than B \& C $(p=0.01 \& p=0.001$ respectively).

Requirement of blood transfusion was a significant factor and more blood transfused in the no preservation group $(p=0.024)$ as shown in

Table-I: Demographic profile of patients in groups.

\begin{tabular}{|c|c|c|c|c|}
\hline & Group "A" $(n=46)$ & Group “B” $(n=70)$ & Group "C" $(n=41)$ & $p$-value \\
\hline \multicolumn{5}{|l|}{ Gender } \\
\hline Male & $26(56.52 \%)$ & $37(52.85 \%)$ & $20(48.78 \%)$ & \multirow{2}{*}{0.774} \\
\hline Female & $20(43.48 \%)$ & $33(47.15 \%)$ & $21(51.22 \%)$ & \\
\hline Mean Age \pm SD & $49.00 \pm 13.362$ & $50.96 \pm 13.702$ & $51.29 \pm 13.316$ & 0.675 \\
\hline BMI & $26.49 \pm 4.259$ & $25.95 \pm 3.981$ & $26.48 \pm 4.004$ & 0.715 \\
\hline LVEF (\%) & $50.11 \pm 8.141$ & $50.49 \pm 9.023$ & $50.88 \pm 10.943$ & 0.929 \\
\hline LVEDD (mm) & $54.87 \pm 7.902$ & $55.63 \pm 6.804$ & $55.38 \pm 8.562$ & 0.443 \\
\hline LVESD (mm) & $43.54 \pm 4.312$ & $44.01 \pm 3.205$ & $45.23 \pm 4.753$ & 0.313 \\
\hline Diabetes & $1(2.17 \%)$ & $6(8.57 \%)$ & $2(4.87 \%)$ & 0.341 \\
\hline Hypertension & $16(34.78 \%)$ & $21(30 \%)$ & $11(26.82 \%)$ & 0.721 \\
\hline Smoking & $5(10.86 \%)$ & $8(11.42 \%)$ & $3(7.31 \%)$ & 0.778 \\
\hline Atrial Fibrillation & $19(41.30 \%)$ & $34(48.57 \%)$ & $18(43.90 \%)$ & 0.733 \\
\hline
\end{tabular}

Table-II: Peri-operative data of patients in groups.

\begin{tabular}{l|c|c|c|c}
\hline & Group “A" (n=46) & Group “B” (n=70) & Group “C” (n=41) & $\boldsymbol{p}$-value \\
\hline CBP TIME (mins) & $135.11 \pm 27.126$ & $136.94 \pm 40.015$ & $120.59 \pm 34.078$ & 0.961 \\
\hline CXT (mins) & $99.17 \pm 22.244$ & $103.79 \pm 30.330$ & $92.49 \pm 25.893$ & 0.639 \\
\hline Mitral valve size (mm) & $27.35 \pm 1.464$ & $28.40 \pm 1.459$ & $29.20 \pm 1.470$ & 0.010
\end{tabular}

$C B P=$ Cardiopulmonary Bypass, $C X T=$ Cross Clamp Time, $p$-valve less than $0.05=$ Statistically significant.

Table-III: Short-term outcome of patients in groups.

\begin{tabular}{l|c|c|c|c}
\hline & Group “A” (n=46) & Group “B” (n=70) & Group “C” (n=41) & $p$-value \\
\hline ICU Stay (Hours) & $28.26 \pm 12.664$ & $33.44 \pm 15.070$ & $40.76 \pm 17.926$ & 0.001 \\
\hline Vent-Time (Hours) & $9.09 \pm 5.807$ & $10.57 \pm 6.854$ & $16.85 \pm 10.056$ & 0.001 \\
\hline Bleeding (mL) & $532.71 \pm 251.187$ & $537.39 \pm 291.416$ & $687.93 \pm 354.894$ & 0.016 \\
\hline Atrial Fibrillation & $9(19.6 \%)$ & $19(27.1 \%)$ & $13(31.7 \%)$ & 0.011 \\
\hline Re-Exploration & $2(4.34 \%)$ & $5(7.14 \%)$ & $8(19.15 \%)$ & 0.036 \\
\hline Death & $1(2.17 \%)$ & $2(2.85 \%)$ & $4(9.75 \%)$ & 0.020 \\
\hline
\end{tabular}

p-value less than $0.05=$ Statistically significant, ICU = Intensive Care Unit.

difference is noted between age, gender, comorbidities like diabetes, hypertension \& smoking, NYHA class \& mitral valve disease type. Pre-
table-II. The pre-operative rhythm was insignificant because on average same number of patients were in sinus vs atrial fibrillation (AF). But post- 
operatively significant rhythm abnormalities like new onset of $\mathrm{AF}$, supra ventricular tachycardia (SVT) \& Ventricular paroxysmal contractions (VPCs) were encountered in the no sub-valvular apparatus group ( $p=0.011)$.

The requirement of post-operative inotropes was significantly more in group $C$ versus group A \& B ( $p=001)$. Post-operative bleeding was more in group C (mean $687.93 \mathrm{ml}$ ) versus group A \& B (mean $532-537 \mathrm{ml}$ ) with $p$-value of 0.016 . There were 2 re-explorations in group A, 5 in group B \& 8 in group $C$ which were statistically significant $(p=0.036)$.

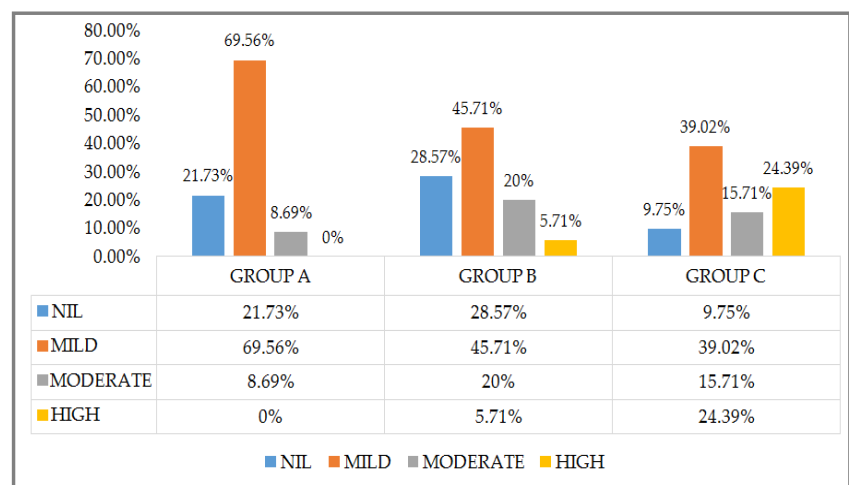

Figure: Inotropic support.

The early mortality was noted to be high in those patients with no sub-valvular apparatus preservation as group $C(n=8)>$ group $B(n=4)$ $>$ group $\mathrm{A}(\mathrm{n}=2)$.

\section{DISCUSSION}

Mitral valve \& its sub-valvular apparatus is a complex anatomical structure with significant effects on Left as well as right ventricular functions ${ }^{1}$. With time it was realized that sub-valvular apparatus is an important determinant in outcome of patient with mitral valve replacement, in reducing early and long term morbidity \& mortality ${ }^{11}$. Different techniques were developed \& practiced in time to time to achieve maximum restoration of normal anatomy \& physiology of mitral valve, like complete preservation without any excision, repositioning of the papillary muscle heads, partial excision of the anterior \& posterior leaflets ${ }^{12}$.
The purpose of our study was to prove that the total sub-valvular apparatus preservation technique had low early mortality \& morbidity in comparison with partial and no preservation. In our study the mortality rate of patients with total sub-valvular apparatus preservation was $2.17 \%$ which is significantly less than those with partial preservation $(2.85 \%)$ \& no preservation $(9.75 \%)$. A study conducted in St. Mary Hospital, London reported mortality of $2.6 \%$ in patients of mitral valve replacement with preservation vs $12.1 \%$ in those without preservation ${ }^{13}$. Another study showed in-hospital mortality of $2.6 \%$ in the preservation group while $8.9 \%$ in the non-preservation group-3 figure showed the inotropic support of patients in all the three groups with requirement of high supports in the no preservation group indicating that patients were in low cardiac output. Similar results were shown by a study conducted in Karachi, where $12.6 \%$ of the patients with preservation required inotropic support while $52.4 \%$ of patients with no preservation needed inotropic support. Therefore, complete and partial preservation was better than no preservation in terms of lesser post-operative complication like low cardiac output, high inotropic support requirement \& mortality ${ }^{14}$.

The reported mortality in different studies conducted on mitral valve replacement with or without preservation demonstrated that main cause of mortality was myocardial pump failure in the postoperative period leading to increased requirement of inotropic support ${ }^{13}$, same cause of high mortality was established in our study because those patients who had no preservation remained in low cardiac output with prolonged ICU stay in comparison with patients of total \& partial preservation. Similar results were reported by Athanasiou et al with significant decrease in requirement of inotropic supports in patients with subvalvular apparatus preservation ${ }^{13}$. Another study showed $7.7 \%$ of patients with preservation required high inotropic support in comparison with $12.6 \%$ of patients in the non-preservation group-3. 
Some researchers believed that total preservation of sub-valvular apparatus prolongs the time of surgery \& implantation of small size prothesis 15 , but findings of our study contradict with the reported evidence as there was no statistically significant difference noted in cardiopulmonary bypass time \& cross clamp time $(p<0.05)$ as shown in table-II. Millers et al reported a significant decrease in mortality of patients with preservation of posterior leaflet \& sub-valvular apparatus $^{16}$. Chowdhury et al in there research showed a significant improvement in the Left Ventricular functions \& reducing the incidence of dilated cardiomyopathy in MVR patients with total preservation of sub-valvular apparatus ${ }^{17}$. Coutinho et al reported mortality of $0.9 \%$ in the preservation group while $1.4 \%$ in the non-preservation group1. Our study results were similar to these studies proving the fact that subvalvular apparatus preservation was an important determinant in better outcome of patients with MVR. Re-exploration rate in our study in group " $\mathrm{A}$ " was $4.3 \%$ while group " $C$ " it was $19.1 \%$, similar results were shown by a study in which $1.8 \%$ of MVR patients with preservation of sub-valvular apparatus were re-explored while $4.0 \%$ in the non-preservation group were re-opened ${ }^{1}$.

In addition to other morbidities in our study, significant decrease in previous AF was noted in the preservation group while in the non-preservation group no such appreciable decrease was noted in patients but also rate of new onset $\mathrm{AF}$ was high in group " $\mathrm{C}$ ". Similar results were reported by a study in which rate of new onset $\mathrm{AF}$ in the preservation group was $19.1 \%$ while in the non-preservation group it was $21.2 \% 1$. Another study showed AF in $38.5 \%$ in preservation group vs $52.7 \%$ in non-preservation group-3.

Postoperative bleeding was another morbidity in our study leading to requirement of RCC transfusion was significantly high in the nonpreservation group; group A < group B < group C. Similar high level of postoperative bleeding was reported in previous resereach ${ }^{3}$. The concept of preservation was highly recommended in the recent era of cardiovascular surgery, not only preservation of the posterior leaflet \& apparatus but the anterior leaflet \& its subvalvular apparatus is also important for the improvement of $\mathrm{LV}$ functions ${ }^{18}$.

\section{CONCLUSION}

From the results of our study it was proved that early mortality \& morbidity was significantly low in those patients with total sub-valvular apparatus preservation. In comparison with the other techniques it requires high surgical experience to avoid the complications of total sub-valvular apparatus preservation. Hence it was suggested that surgeons should know about the different techniques of preservation and its expected complication.

\section{CONFLICT OF INTEREST}

This study has no conflict of interest to be declared by any author.

\section{REFERENCES}

1. Coutinho GF, Bihun V, Correia PE, Antunes PE, Antunes MJ. Preservation of the subvalvular apparatus during mitral valve replacement of rheumatic valves does not affect long-term survival. Eur J Cardio Thorac Surg 2015; 48: 861-67.

2. Gunnal SA, Wabale RN, Farooqui MS. Morphological study of chordae tendinae in human cadaveric hearts. Heart Views 2015; 16(1): 1-5.

3. Ghavidel AA, Mirmesd Y, Sharifi M, Nakhaeizadeh AR, Omrani GR. The impact of subvalvular apparatus preservation on prosthetic vavle dysfunction during mitral valve replacement. Res Cardiovasc Med 2013; 2(1): 55-61.

4. Kessel SM, Hawkins RB, Yarboro LT, Ailawadi G. Total chordal sparing mitral valve replacement in rheumatic disease: a word of caution. Ann Thorac Surg 2017; 104: 47-48.

5. Zilla P, Koshy J, Brink J, Human P. Mitral valve replacement for rheumatic heart disease in Southern Africa. J Cardiothorac Surg 2013; 8(1): 4-5.

6. Chen L, Chen B, Hao J, Wang X, Ma R, Cheng W. Complete preservation of the mitral valve apparatus during mitral valve replacement for rheumatic mitral regurgitation in patients with an enlarged left ventricular chamber. Heart Surg Forum 2013; 16: 137-43.

7. Dinara B. Toktosunova. Strategy of complete preservation of the subvalvular structures in the mitral valve replacement. Heart Vessels Transplant 2019; 3(1): 123-30.

8. Gomes OM, Gomes ES, Santana FGP. New technical approach for crossed papillopexy in mitral valve replacement surgery: short term results. Braz J Cardiovasc Surg 2005; 20: 340-45.

9. Alsaddique AA. Mitral valve replacement with the preservation of the entire valve apparatus. Rev Bras Cir Cardiovasc 2007; 22: 218-23.

10. Puig LB, Gaiotto FA, Oliveira JL, Pardi MM, Bacal F, Mady C, et al. Mitral valve replacement and remodeling of the left ventricle in dilated cardiomyopathy with mitral regurgitation: initial results. Arq Bras Cardiol 2002; 78(2): 224-29. 
11. Lillehei CW, Levy MJ, Bonnabeau RC. Mitral valve replacement with preservation of papillary muscles and chordaetendineae. J Thorac Cardiovasc Surg 1964; 47: 532-43.

12. Guo Y, He S, Wang T, Chen Z, Shu Y. Comparison of modified total leaflet preservation, posterior leaflet preservation and no leaflet preservation techniques in mitral valve replacement- a retrospective study. J Cardiothorac Surg 2019; 14: 102.

13. Athanasiou T, Chow A, Roa C, Aziz O, Siannis F, Ali A, Darzi A, Wells F. Preservation of the mitral valve apparatus: evidence synthesis and critical reappraisal of surgical techniques. Eur J Cardiothorac Surg 2008; 33: 391-401.

14. Zakai SB, Khan SUR, Rabbi F, Tasneem H. Effects of mitral valve replacement with and without chordal preservation on cardiac function: early and mid- term results. J Ayub Med Coll Abbottabad 2010; 22: 91-96.
15. Kisamori E, Otani S, Yamamoto T, Nishiki M, Yamada $Y$, Matsumoto T. Mitral valve repair versus replacement with preservation of the entire subvalvular apparatus. Gen Thorac Cardiovasc Surg 2018; 1039-2.

16. Miller DW, Johnson DD, Ivey TD. Does preservation of the posterior chordae tendineae enhance survival during mitral valve replacement?. Ann Thorac Surg 1979; 28: 22-27.

17. Chowdhury UK, Kumar AS, Airan B, Mittal D, Subramaniam G, Prakash $\mathrm{R}$, et al. Mitral valve replacement with and without chordal preservation in a rheumatic population: serial echocardiographic assessment of LV size and function. Ann Thorac Surg 2005; 79: 1926-33.

18. Ozdemir AC, Emrecan B, Baltalarli A. Bileaflet versus posterior leaflet only preservation inmitral valve replacement. Tex Heart Inst J 2014; 41: 165-69. 